\title{
Effects of hadronic repulsive interactions on the fluctuations of conserved charges
}

\author{
Somenath Pal, ${ }^{1, *}$ Guruprasad Kadam $\odot,{ }^{2, \dagger}$ Hiranmaya Mishra, ${ }^{3, \star}$ and Abhijit Bhattacharyya $\odot^{1, \S}$ \\ ${ }^{1}$ Department of Physics, University of Calcutta, 92, A.P.C. Road, Kolkata 700009, India \\ ${ }^{2}$ Department of Physics, Shivaji University, Kolhapur, Maharashtra 416004, India \\ ${ }^{3}$ Theory Division, Physical Research Laboratory, Navarangpura, Ahmedabad 380 009, India
}

(Received 22 October 2020; accepted 19 February 2021; published 15 March 2021)

\begin{abstract}
We investigate the effect of repulsive interaction between hadrons on the fluctuations of the conserved charges. We calculate the baryon, the electric charge, and the strangeness susceptibilities within the ambit of hadron resonance gas model extended to include the short range repulsive interactions. The repulsive interactions are included through a mean-field approach where the single particle energy gets modified due to mean-field interactions between hadrons proportional to the number density of hadrons. We assume different mean-field interactions for mesons and baryons. It is shown that the repulsive interactions play a very crucial role to describe hadronic matter near transition temperature. We also show that in order to consistently describe higher order conserved charge fluctuations mesonic repulsive interactions cannot be neglected. Further, we demonstrate that the repulsive interaction of baryons is essential to describe the lattice simulation results at finite baryon chemical potential for higher order fluctuations.
\end{abstract}

DOI: 10.1103/PhysRevD.103.054015

\section{INTRODUCTION}

Studies of strongly interacting matter at high temperatures and/or densities have been a vibrant area of research for several decades. At such high temperatures and/or densities, the effective degrees of freedom of the strongly interacting matter are colored quarks and gluons whereas at low temperatures and/or densities these are colorless hadrons. There are several ongoing and upcoming experiments with ultrarelativistic heavy-ion collision which are recreating such phases of strongly interacting matter. The experimental programs, Large Hadron Collider at Geneva and Relativistic Heavy Ion Collider at Brookhaven, have already provided a plethora of data. The upcoming facilities like Facility for Antiproton and Ion Research and Nuclotron-based Ion Collider fAcility are expected to shed more light in this area of research.

One of the main objectives of these explorations is to understand the phase diagram of strongly interacting matter. At high temperature and at vanishing or small

\footnotetext{
*somenathpal1@gmail.com

†uruprasadkadam18@gmail.com

hm@prl.res.in

$\S$ abhattacharyyacu@gmail.com
}

Published by the American Physical Society under the terms of the Creative Commons Attribution 4.0 International license. Further distribution of this work must maintain attribution to the author(s) and the published article's title, journal citation, and DOI. Funded by SCOAP ${ }^{3}$. values of baryon chemical potential, the transition between the hadronic matter and the quark-gluon matter is established to be a crossover [1,2]. On the other hand, at low temperature and high baryon densities, it is generally expected that such a transition is possibly a first order phase transition [3-8]. Therefore, in the phase diagram of strong interaction in the temperature and baryon chemical potential plane, one expects a first order line ending in a critical point [9-12]. The search for this elusive critical end point (CEP) of strong interaction has become an extremely active field of research since last few years, although its existence as a fundamental property of strong interaction still remains to be confirmed experimentally. The CEP is typically characterized by large fluctuations of the static thermodynamic quantities due to large correlation length. One of the crucial realizations in this context has been the fact that the measurement of the moments of the conserved quantities, viz., baryon number, charge, and strangeness, can be accessible in heavy-ion collisions experiments and can be linked to susceptibilities that can be computed in QCD based calculations. In order to confirm, whether the thermalized system, produced in heavy-ion collisions, has passed through the quark-hadron phase transition, it is necessary to make theoretical calculations of the fluctuations of conserved charges from both sides of the phase transition line.

Though quantum chromodynamics (QCD) is the theory of strong interactions, the traditional perturbative methods of field theory cannot be applied in the case of temperature/ density of interest. Lattice QCD (LQCD) has been the 
principle tool to understand the equilibrium phase structure of strongly interacting matter and the fluctuations of conserved charges have been extensively studied [1,13-27]. Despite its success at zero baryon density, LQCD calculations have limited applicability at finite baryon density. Hence, in the finite density region of QCD phase diagram, one needs to take resort to effective models of QCD which preserve some essential properties of QCD at a given energy scale. For instance, Polyakov-Nambu-Jona-Lasinio model [28-43], hadron resonance gas (HRG) model [44-54], polyakov-quark-meson model [55-58], chiral perturbation theory [59], etc. have been rather successful in describing different parts of the finite density region of the phase diagram. These models have also been found to reproduce the lattice data at zero density quite successfully [37,38]. Apart from investigations of the phase structure of strong interaction in such effective models, there have been significant progress in this field using functional methods for first principle QCD like functional renormalization group approach [60] and Dyson-Schwinger approach [61].

Hadron resonance gas model is a low temperature statistical thermal model describing hadronic phase of QCD. This model is based on S-matrix formulation of statistical mechanics [62]. In the relativistic virial expansion of the partition function, the interactions are manifested in the form of phase shifts in the two particle scattering. If such scattering occurs through exchange of a narrow resonance state, then the interacting partition function just becomes a noninteracting partition function with the additional contribution from the exchanged resonance [63-65]. Indeed, it turns out that the nonresonant terms cancel out in the estimation of certain thermodynamic quantities. Thus, in the HRG model, the thermodynamic quantities of low temperature hadronic matter can be obtained from the partition function which contains all relevant degrees of freedom of the confined QCD phase and implicitly includes the (attractive) interactions that result in resonance formation.

Despite its success, it was soon realized that noninteracting HRG model is not sufficient to describe hadronic matter, particularly, near quark-hadron transition temperature, $T_{c}$. As temperature increases, gas density increases and at high temperature the assumption of dilute gas approximation, a principal assumption of HRG model, need not be valid. The hadronic repulsive interaction becomes increasingly important as one approaches the critical temperature. The validity of HRG model can only be checked by confronting its equation of state (EOS) with LQCD simulations. Various studies have confronted ideal HRG EOS with lattice results and found reasonable agreement all the way up to $T_{c}$ except for interaction measure [66]. Later studies found good agreement with the lattice data for trace anomaly as well, when continuous Hagedorn states are included in HRG model $[67,68]$. However, recent studies have shown that commonly performed comparisons of ideal HRG model with LQCD simulations and heavy-ion data may lead to misconceptions which might further render wrong conclusions and it is necessary to take into account short range repulsive interactions among the hadrons as they play a crucial role in the thermodynamics of hadron gas [69].

In the present investigation, we would like to estimate conserved charge fluctuations within the ambit of an interacting HRG model that includes the repulsive interactions. Equilibrium fluctuations, of conserved charges, has been studied using the ideal HRG model [70-73] (See Ref. [74] for a review). This analysis showed that the lower order cumulants are reasonably well reproduced but higher order cumulants show significant deviation. One possible way to include such repulsive interaction is through the van der Walls excluded volume approach as in Refs. [75,76]. Another approach has been to include repulsive interaction through a repulsive density-dependent mean field [77-80]. Such a mean-field HRG (MFHRG) model has been used to compute fluctuations in the net baryon number and the strangeness-baryon correlation at vanishing chemical potentials [79]. This was also considered for hadron thermodynamics and transport properties [80] of hadronic matter. Specifically, we wish to estimate in the present work, the second and the fourth order fluctuations for baryon number $(B)$, electric charge $(Q)$, and strangeness $(S)$ quantum numbers using MFHRG. We also estimate the ratios as well as the differences of second and fourth order fluctuations. While the ratios of fluctuations are important so as to remove the effect of system volume, differences of fluctuations remove the effect of mass spectrum included in HRG model and one can distill the effect of only repulsive interactions on the fluctuations. It may further be noted that differences of susceptibilities are also indicators of deconfinement phase transition [81]. We estimate these susceptibilities both at vanishing and finite baryon densities.

We have organized the paper as follows. In Sec. II, we discuss the thermodynamics of relativistic meanfield hadron resonance gas model and define the susceptibilities. Results of cumulants are discussed in Sec. III. Finally, in Sec. IV, we summarize our findings and present our conclusions.

\section{HADRON RESONANCE GAS MODEL WITH A REPULSIVE MEAN-FIELD INTERACTION}

Thermodynamic properties of ideal hadron resonance gas model can be derived from the grand canonical partition function given by

$$
\begin{aligned}
\ln \mathcal{Z}\left(T, \mu_{B}, \mu_{Q}, \mu_{S}, V\right)= & \sum_{\text {mesons }} \ln \mathcal{Z}\left(T, \mu_{Q}, \mu_{S}, V\right) \\
& +\sum_{\text {baryons }} \ln \mathcal{Z}\left(T, \mu_{B}, \mu_{Q}, \mu_{S}, V\right),
\end{aligned}
$$


where $\mu_{B}, \mu_{Q}$, and $\mu_{S}$ are the chemical potentials corresponding to baryon number, electric charge, and strangeness, respectively. The pressure can be obtained from the partition function as

$$
\begin{aligned}
P & \left(T, \mu_{B}, \mu_{Q}, \mu_{S}, V\right) \\
& =\lim _{V \rightarrow \infty} \frac{T}{V} \ln \mathcal{Z}\left(T, \mu_{B}, \mu_{Q}, \mu_{S}, V\right) .
\end{aligned}
$$

Partition function of $i$ th hadronic species is

$$
\ln \mathcal{Z}\left(T, V, \mu_{a}\right)={ }_{ \pm} V \int d \Gamma_{a} \ln \left[1 \pm e^{\left.-\frac{\left(E_{a}-c_{i} \mu_{i}\right)}{T}\right]}\right.
$$

where, for the $i$ th species of hadrons, $c_{i} \equiv\left(B_{i}, Q_{i}, S_{i}\right)$ corresponds to the respective conserved charges and $\mu_{i} \equiv$ $\left(\mu_{B}, \mu_{Q}, \mu_{S}\right)$ is the corresponding chemical potential. Also, $d \Gamma_{a} \equiv \frac{g_{a} d^{3} p}{(2 \pi)^{3}}$ with $g_{a}$ being the spin degeneracy factor and $E_{a}=\sqrt{p^{2}+m_{a}^{2}}$ is the relativistic single particle energy with mass $m_{a}$. Upper (lower) sign corresponds to fermions (bosons).

Ideal HRG model can be extended by including short range repulsive interactions between hadrons. These repulsive interactions can be treated in mean-field approach where the single particle energies $\epsilon_{a}$ get shifted by the mean-field repulsive interaction as $[77,78]$

$$
\varepsilon_{a}=\sqrt{p^{2}+m_{a}^{2}}+U(n)=E_{a}+U(n),
$$

where $n$ is the total hadron number density. The potential energy $U$ represents repulsive interaction between hadrons and it is taken to be function of total hadron density $n$. For an arbitrary interhadron potential $V(\mathbf{r})$, the potential energy $U$ is given by

$$
U(n)=K n,
$$

where $K$ is a phenomenological parameter given by the spatial integration of the interhadron potential $V(\mathbf{r})$.

In the present investigation, we assume different repulsive interaction parameter for baryons and mesons. We denote the mean-field parameter for baryons $(B)$ and antibaryons $(\bar{B})$ by $K_{B}$, while for mesons we denote it by $K_{M}$. Thus, for baryons (antibaryons),

$$
U\left(n_{B\{\bar{B}\}}\right)=K_{B} n_{B\{\bar{B}\}}
$$

and for mesons

$$
U\left(n_{M}\right)=K_{M} n_{M}
$$

Few comments in this context may be of relevance. In the present investigation, we have assumed the same repulsive interactions between all the baryonic species which perhaps is an oversimplification. For example, the interaction between nucleon hyperon or hyperon hyperon which has been studied both in lattice and in chiral effective theories indicates that while the interactions are dominantly repulsive, there are differences as compared to nucleon nucleon interactions. However, we do not have sufficient information about baryon baryon interaction to have a more sophisticated mean-field model. Furthermore, the baryons can interact with antibaryons and one might expect that this may also be significant. However, at least within the Boltzmann approximation for the baryonic distribution functions, the contribution to the pressure from such interactions will be independent of the chemical potential and hence will not contribute to the susceptibilities. Similarly, one does not have the scope of introducing the baryon/antibaryon and meson repulsive interaction within the mean-field model. We will continue with the simple approximation of including the same repulsive interaction between baryon and among mesons given by Eqs. (6) and (7) similar to Refs. [79,80]. This is in contrast to the approach of including hadronic repulsive interaction through a van der Walls excluded volume approach $[82,83]$ where the repulsive interaction among all the hadrons is taken into account.

The total hadron number density is

$$
n(T, \mu)=\sum_{a} n_{a}=n_{B}+n_{\bar{B}}+n_{M},
$$

where $n_{a}$ is the number density of ath hadronic species. Also, $n_{B}, n_{\bar{B}}$, and $n_{M}$ are total baryon, antibaryon, and meson number densities, respectively. For baryons,

$$
n_{B}=\sum_{a \in B} \int d \Gamma_{a} \frac{1}{e^{\frac{\left(E_{a}-\mu_{\mathrm{eff}, B}\right)}{T}+1},}
$$

where $\mu_{\mathrm{eff}, B}=c_{i} \mu_{i}-K_{B} n_{B}$ and $c_{i}=\left(B_{i}, Q_{i}, S_{i}\right), \mu_{i}=$ $\left(\mu_{B}, \mu_{Q}, \mu_{S}\right)$. The sum is over all the baryons. Similarly, the number density of antibaryons is

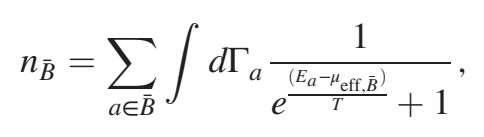

where $\mu_{\text {eff, } \bar{B}}=\bar{c}_{i} \mu_{i}-K_{B} n_{\bar{B}}$. Note that repulsive mean-field parameter is same for baryons as well as antibaryons. For mesons,

$$
n_{M}=\sum_{a \in M} \int d \Gamma_{a} \frac{1}{e^{\frac{\left(E_{a}-\mu_{\mathrm{eff}, M)}\right.}{T}}-1},
$$

where $\mu_{\mathrm{eff}, M}=c_{i} \mu_{i}-K_{M} n_{M}$ and the sum is over all the mesons.

Equations (9)-(11) are actually self-consistent equations for number densities which can be solved numerically. The expressions for the pressures of baryons and mesons are then given, respectively, as 


$$
\begin{aligned}
P_{B\{\bar{B}\}}(T, \mu)= & T \sum_{a \in B\{\bar{B}\}} \int d \Gamma_{a} \ln \left[1+e^{-\left(\frac{E_{a}-\mu_{\mathrm{eff}}\left\{\bar{\mu}_{\mathrm{eff}}\right\}}{T}\right)}\right] \\
& -\phi_{B\{\bar{B}\}}\left(n_{B\{\bar{B}\}}\right), \\
P_{M}(T, \mu)=- & T \sum_{a \in M} \int d \Gamma_{a} \ln \left[1-e^{\left.\frac{\left(E_{a}-\mu_{\mathrm{eff}, M}\right)}{T}\right]}-\phi_{M}\left(n_{M}\right),\right.
\end{aligned}
$$

where

$$
\phi_{B}\left(n_{B\{\bar{B}\}}\right)=-\frac{1}{2} K_{B} n_{B\{\bar{B}\}}^{2}
$$

and

$$
\phi_{M}\left(n_{M}\right)=-\frac{1}{2} K_{M} n_{M}^{2}
$$

Thermodynamic quantities can be readily calculated by taking appropriate derivative of the partition function or equivalently of the pressure.

The nth-order susceptibility is defined as

$$
\chi_{i}^{n}=\frac{\partial^{n}\left(P\left(T, \mu_{i}\right) / T^{4}\right)}{\partial\left(\frac{\mu_{i}}{T}\right)^{n}},
$$

where $\mu_{i}$ is the chemical potential for conserved charge $c_{i}$. In this work, we will take $i$ to be baryon number $(B)$, electric charge $(Q)$, and strangeness $(S)$. The $n=1,2,3,4$ susceptibilities are related to the mean, variance, skewness, and kurtosis of the distribution of the conserved charges.

Before proceeding further, let us discuss some approximate expressions for the pressure and the number densities and hence on the susceptibilities which are useful to analyze the behavior of susceptibilities with temperature and/or chemical potentials. One can expand the logarithm in the expression for (noninteracting HRG) pressure in powers of fugacity so that the baryonic pressure in Eq. (12) can be written as (with $\beta=T^{-1}$ )

$$
\begin{aligned}
\frac{P_{B\{\bar{B}\}}}{T^{4}}= & \sum_{a \in B\{\bar{B}\}} \frac{g_{a}}{2 \pi^{2}}(\beta m)^{2} \sum_{l=1}^{\infty}(-1)^{l+1} l^{-2} K_{2}\left(\beta l m_{a}\right) z^{l} \\
& +\frac{K_{B} T^{2}}{2}\left(\frac{n_{B\{\bar{B}\}}}{T^{3}}\right)^{2} .
\end{aligned}
$$

In the above, we have defined the fugacity as $z=$ $\exp \left(\beta \mu_{\text {eff }}\right)$ and $K_{2}$ is the Bessel function. It can be easily shown that as long as $\beta\left(m_{a}-\mu_{\text {eff }}\right) \gtrsim 1$, the contribution to the pressure $P_{B\{\bar{B}\}}^{i d}$ can be approximated by the leading term, i.e., $l=1$ in the summation which, in fact, corresponds to Boltzmann approximation. In this limit, the pressure from the baryons becomes

$$
\begin{aligned}
\frac{P_{B\{\bar{B}\}}}{T^{4}}= & \sum_{a \in B} \frac{g_{a}}{2 \pi^{2}}\left(\beta m_{a}\right)^{2} K_{2}\left(\beta m_{a}\right) \\
& \times \exp \left(\beta \mu_{\text {eff }}^{a}\right)+\frac{K_{B} T^{2}}{2}\left(\frac{n_{B\{\bar{B}\}}}{T^{3}}\right)^{2} .
\end{aligned}
$$

In a similar approximation, the number density for baryons can be written as

$$
\frac{n_{B}}{T^{3}}=\sum_{a \in B} \frac{g_{a}}{2 \pi^{2}}(\beta m)^{2} K_{2}\left(\beta m_{a}\right) e^{\beta \mu_{\mathrm{eff}}^{a}} .
$$

Thus, the interacting gas pressure in the Boltzmann approximation can be written as

$$
P_{B\{\bar{B}\}}=T n_{B\{\bar{B}\}}+\frac{K_{B}}{2} n_{B\{\bar{B}\}}^{2} .
$$

One can further approximate for the number densities given in Eq. (19) by noting that, for temperatures below the QCD transition temperatures such that $n_{B}\left(n_{\bar{B}}\right)$ are small, one can expand the exponential $\exp \left(\mu_{\text {eff }}\right) \simeq$ $\exp \left(c_{i} \mu_{i}\right)\left(1-\beta K_{B} n_{B}\right)$. This leads to $n_{B}=n_{B}^{i d} /\left(1+n_{B}^{i d}\right)$. Here $n_{B}^{i d}$ is the number density without any repulsive interaction [Eq. (21)], i.e.,

$$
\frac{n_{B}^{i d}}{T^{3}}=\sum_{a \in B} \frac{g_{a}}{2 \pi^{2}}\left(\beta m_{a}\right)^{2} K_{2}\left(\beta m_{a}\right) \exp \left(\beta c_{a}^{i} \mu_{i}\right) .
$$

The pressure due to baryons can then be approximated as

$$
P_{B}=T n_{B}^{i d}-\frac{K_{B}}{2}\left(n_{B}^{i d}\right)^{2} .
$$

A similar expression can be obtained for antibaryon pressure. As may be noted, the effect of the densitydependent repulsive interaction essentially lies in reducing the pressure at finite densities.

The total pressure from baryons and antibaryons can then be written as

$$
\begin{aligned}
\frac{P_{B}+P_{\bar{B}}}{T^{4}} \simeq & \sum_{a \in B} F_{a}\left(\beta m_{a}\right) \cosh \left(\beta c_{a}^{i} \mu_{i}\right) \\
& -\frac{K_{B} T^{2}}{2}\left(\sum_{a} G_{a}\left(\beta m_{a}, \beta \mu_{Q}, \beta \mu_{s}\right)\right)^{2} e^{2 \beta \mu_{B}} \\
& -\frac{K_{B} T^{2}}{2}\left(\sum_{a} G_{a}\left(\beta m_{a},-\beta \mu_{Q},-\beta \mu_{s}\right)\right)^{2} e^{-2 \beta \mu_{B}} .
\end{aligned}
$$

Here we have defined the chemical potentialindependent function $F_{a}\left(\beta m_{a}\right)$ as

$$
F_{a}\left(\beta m_{a}\right)=\frac{g_{a}}{\pi^{2}}\left(\beta m_{a}\right)^{2} K_{2}\left(\beta m_{a}\right)
$$


and the baryon chemical potential-independent function as

$$
\begin{aligned}
G_{a}\left(\beta m_{a}, \beta \mu_{Q}, \beta \mu_{s}\right)= & \frac{g_{a}}{2 \pi^{2}}\left(\beta m_{a}\right)^{2} K_{2}\left(\beta m_{a}\right) \\
& \times \exp \left(Q_{a} \beta \mu_{Q}+S_{a} \beta \mu_{s}\right) .
\end{aligned}
$$

In a similar manner, for the mesons, in the Boltzmann approximation, and with $\beta K_{m} n_{m} \leq 1$, we have

$$
\frac{P_{M}}{T^{4}} \simeq \sum_{a \in M} \frac{n_{a}^{i d}}{T^{3}}-\frac{1}{2}\left(K_{M} T^{2}\right)\left(\frac{n_{a}^{i d}}{T^{3}}\right)^{2}
$$

with

$$
n_{a}^{i d}=\frac{g_{a}}{2 \pi^{2}} K_{2}\left(\beta m_{a}\right) \exp \left(\beta \mu_{a}\right) .
$$

The total pressure $P=P_{B}+P_{\bar{B}}+P_{M}$ is thus given approximately by the sum of Eqs. (23) and (26). These approximate expressions for pressure have interesting consequences. First, in the context of baryonic susceptibilities, the odd order susceptibilities will be small for small chemical potential and will vanish for zero baryonic chemical potential. Further, for the even order baryonic susceptibilities, e.g., $\chi_{B}^{4}$ and $\chi_{B}^{2}$ will be identical but for the repulsive interaction term. Indeed, the difference between these is given approximately as

$$
\chi_{B}^{2}-\chi_{B}^{4} \simeq 12 \frac{K_{B} T^{2}}{2}\left(\beta^{3} n_{B}^{i d}\right)^{2} .
$$

Thus, while the difference between the higher(even) order and lower(even) order baryonic susceptibilities will vanish for ideal HRG, it will not vanish when there is mean-field repulsive terms. In the following, we shall discuss the results where we take the actual Fermir-Dirac or BoseEinstein statistics for the hadrons and solve for the selfconsistent equations for the number densities for the estimation of susceptibilities. However, as we shall observe, the above assertions made with the approximate expressions for the pressure remain valid.

\section{RESULTS AND DISCUSSION}

In this section, we are going to discuss the results of susceptibilities of different conserved quantities calculated from the MFHRG model. We will compare our results with those obtained from LQCD; namely with Ref. [84] for vanishing chemical potential and with Ref. [85] for nonvanishing chemical potentials. Fluctuations of conserved charges like net baryon number, electric charge, strangeness are useful indicators of thermalization and hadronization of matter produced in ultrarelativistic heavy-ion collision [37,38,86-92]. Large fluctuations in various thermodynamic quantities are important signatures of existence of CEP in the phase diagram. To estimate the different thermodynamic quantities, we have taken all the hadrons and resonances up to $3 \mathrm{GeV}$ listed in particle data review [93]. The only parameters in our model are $K_{M}$ and $K_{B}$ as mentioned in the previous section. We choose three different representative values for meson mean-field parameter, viz., $K_{M}=0,0.1$, and $0.15 \mathrm{GeV} \cdot \mathrm{fm}^{3}$, while we fix baryon mean-field parameter $K_{B}=0.45 \mathrm{GeV} \cdot \mathrm{fm}^{3}[79,80]$.

In Fig. 1, we plot the second and fourth order baryon number susceptibilities and compare them with the recent lattice QCD results of Ref. [94]. In the absence of any repulsion, the susceptibilities, calculated in the HRG model,
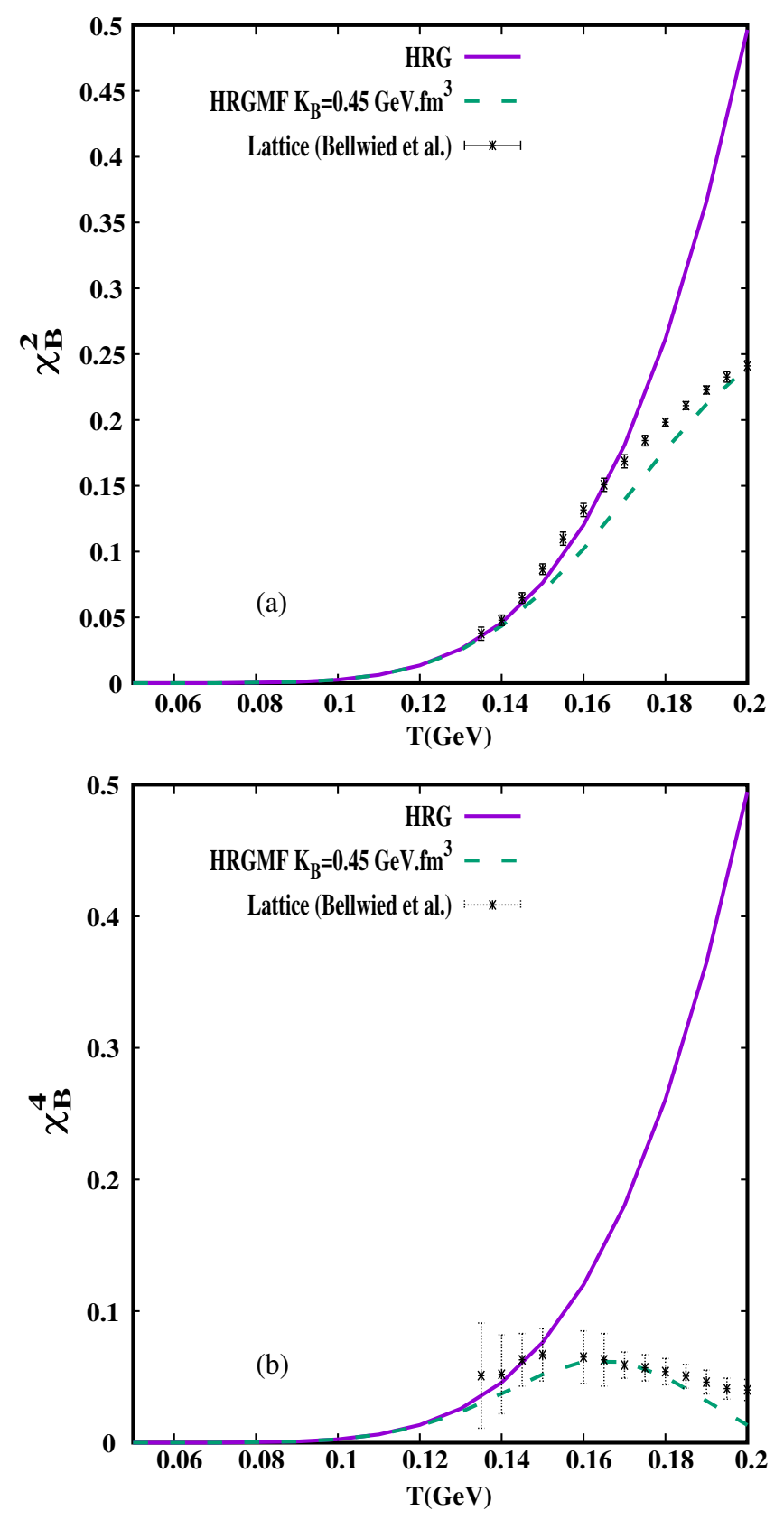

FIG. 1. Baryon number susceptibilities of different orders. This result is independent of $K_{m}$. The lattice data of both $\chi_{B}^{2}$ and $\chi_{B}^{4}$ are taken from Ref. [94]. 
increase monotonically with temperature. However, as the interaction, among the baryons, is switched on through $K_{B}$, the susceptibilities (especially $\chi_{B}^{4}$ ) show nonmonotonic behavior. We note that HRG model reproduces the LQCD results up to a temperature of $T=160 \mathrm{MeV}$ after which it deviates. On the other hand, MFHRG provides a very good qualitative and quantitative description. The broad bump in $\chi_{B}^{4}$ [Fig. 1(b)], as obtained in the lattice simulations, is very well reproduced in MFHRG model. It may be observed that while the behavior of $\chi_{B}^{2}$ is only qualitatively matches with the lattice results, the agreement of the same for $\chi_{B}^{4}$ is both qualitative and quantitative. The repulsive interaction reduces the baryonic susceptibility at higher temperatures. This may be expected from the approximate expressions for pressure given in Eq. (23). This also explains that the reduction is more for the higher order susceptibility. Note that mesons do not contribute to $\chi_{B}$ and hence the results are independent of the value of $K_{m}$. Since all the baryons have baryon number one, the numerical values of $\chi_{B}^{2}$ and $\chi_{B}^{4}$ are same for HRG. However, this is not true for the interacting (MFHRG) scenario. Agreement of MFHRG with LQCD emphasizes the role of repulsive interaction in the thermodynamics of hadron gas especially at higher temperature. Recently, similar studies have observed that if we switch off the repulsive interactions between mesons and include van der Waals parameters characterizing repulsive and attractive interaction between baryons, the resulting model turns out to be in better agreement with the lattice data [69]. Thus, ideal HRG is insufficient to describe higher order susceptibilities and the agreement with the results from mean-field HRG, which takes into account repulsive interactions, indicates that these interactions cannot be neglected in the studies which are being carried out to probe quark-hadron phase transition as well as QCD critical point.

Figure 2 shows second and fourth order electric charge susceptibilities as calculated in HRG, MFHRG, and lattice QCD. The lattice results from $\chi_{Q}^{2}$ are taken from Ref. [95], while the same for $\chi_{Q}^{4}$ is taken from Ref. [96]. As in the case of baryon number susceptibilities, the electric charge susceptibilities increase monotonically with temperature for HRG. However, the numerical values of $\chi_{Q}^{2}$ and $\chi_{Q}^{4}$ are not same for HRG, which is unlike baryon number susceptibilities. We note that there is no qualitative and almost no quantitative difference between results obtained from HRG and MFHRG model when $K_{M}=0$ except at high temperature. The reason is that the dominant contribution to $\chi_{Q}^{n}$ arises from charged mesons for which there is no repulsive interactions for $K_{m}=0$. But when we switch on mean-field interactions for mesons, the MFHRG model becomes closer to the LQCD results for the susceptibility $\chi_{Q}^{2}$. Reasonable quantitative agreement is achieved for $K_{M}=0.1 \mathrm{GeV} \cdot \mathrm{fm}^{3}$. In case of $\chi_{Q}^{4}$, MFHRG overestimates the susceptibility for all the three choices of $K_{M}$. Nonetheless, qualitative agreement can be
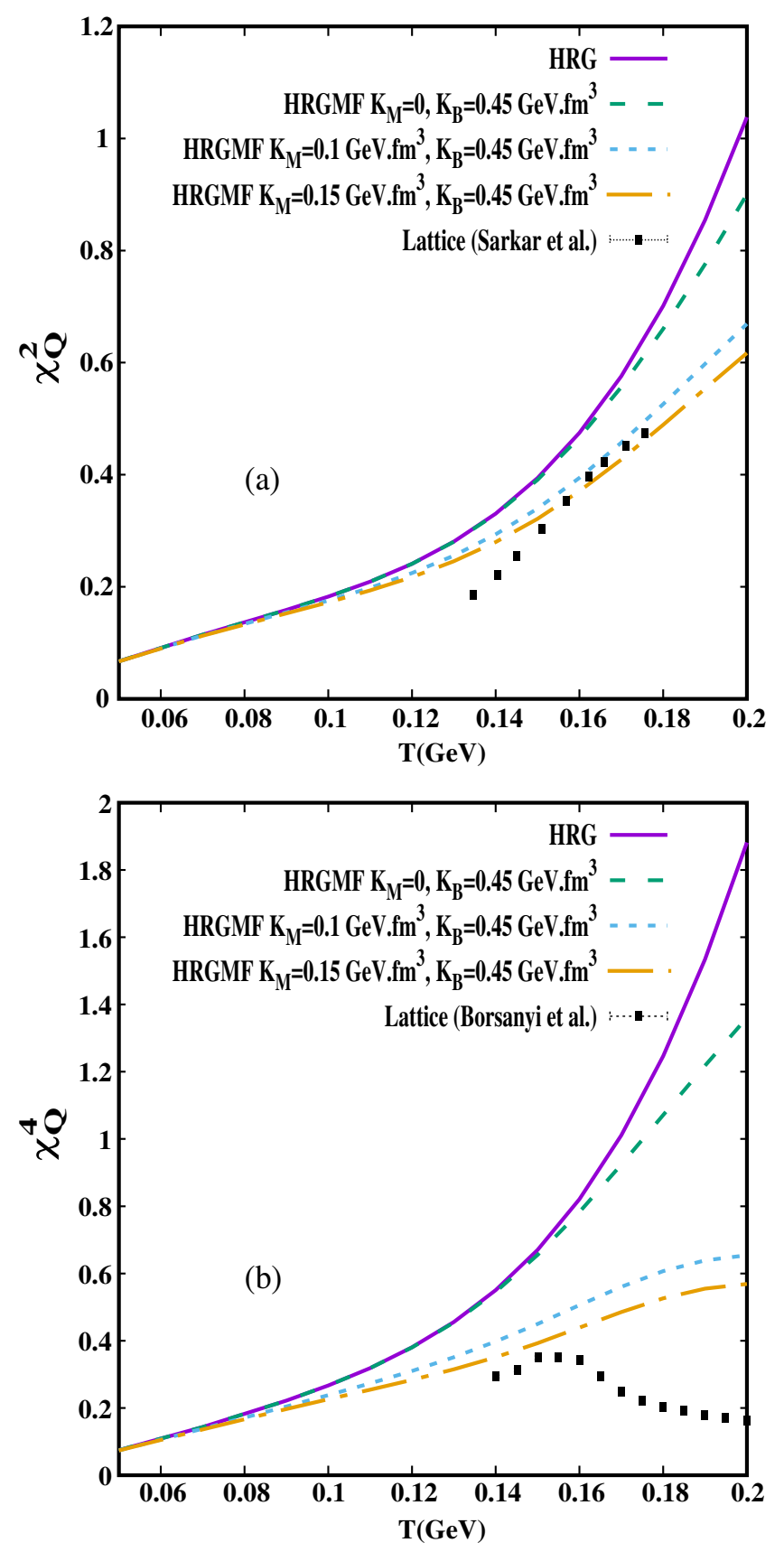

FIG. 2. Charge susceptibilities of different orders. The lattice data of $\chi_{Q}^{2}$ are taken from Ref. [95] and the lattice data of $\chi_{Q}^{4}$ are taken from [96].

seen for higher values of $K_{M}$ which also emphasizes the important role of repulsive interactions among mesons.

In Fig. 3, we have plotted second and fourth order strangeness susceptibilities. Here, the lattice results for $\chi_{S}$ and $\chi_{S}^{4}$ are taken from Ref. [96]. As may be observed, the HRG results seem to be more close to the lattice results for $\chi_{S}^{2}$ compared to the MFHRG results. On the other hand, for $\chi_{S}^{4}$, while HRG results match up to $T=160 \mathrm{MeV}$, for higher temperatures HRG shows a monotonic increase with temperature while lattice results seem to flatten out around 

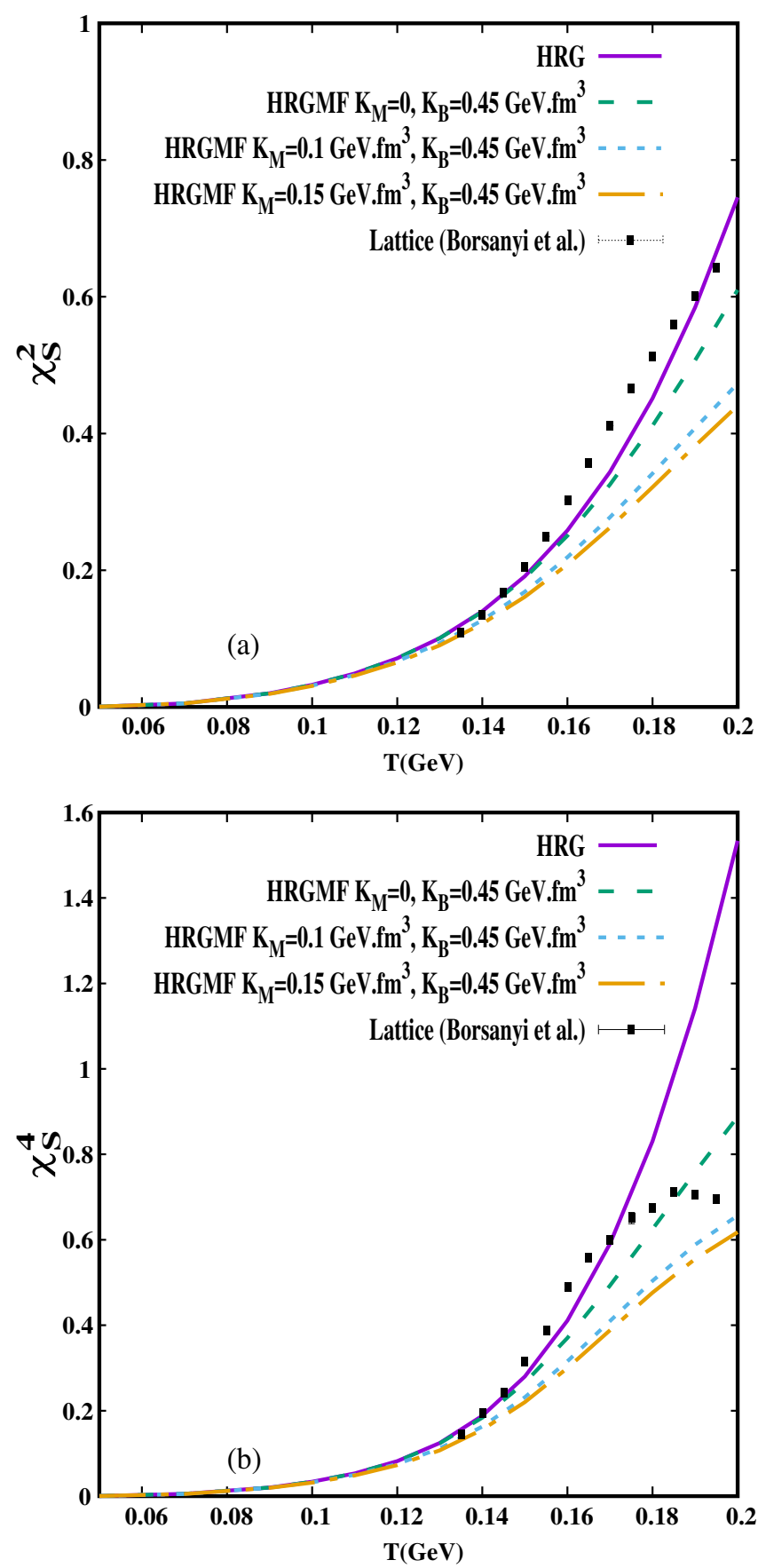

FIG. 3. Strangeness susceptibilities of different orders. The lattice data of both $\chi_{S}^{2}$ and $\chi_{S}^{4}$ are taken from Ref. [96].

$T=180 \mathrm{MeV}$. Such behavior is qualitatively reproduced by MFHRG model. Further, it is also observed that the repulsive interactions seem to underestimate strangeness susceptibilities. Previous studies have also observed similar behavior of strangeness susceptibilities as well as correlators involving strangeness [97]. This observation can be attributed to the unknown strange hadronic states that are not included in the hadronic mass spectrum. In fact, inclusion of these unknown states has been found to improve HRG model estimations [98].
Figure 4 shows the ratios of second and fourth order susceptibilities of baryon, charge, and strangeness. These ratios are related to the moments of conserved charge fluctuations and hence they are important in the context of heavy-ion collision experiments. In the MFHRG model, as mentioned earlier, in the Boltzmann approximation (and in the limit of $\beta U \ll 1$ ), the susceptibility $\chi_{B}^{n}$ can be approximated as

$$
\chi_{B}^{n}=\left(\chi_{B}^{i d}\right)^{n}-2^{n} \beta^{4} K_{B}\left(n_{B}^{i d}\right)^{2},
$$

where $\left(\chi_{B}^{i d}\right)^{n}$ is the nth order noninteracting susceptibility. We note from the above equation that when repulsive interactions are switched off $\left(K_{B}=0\right)$ one gets $\frac{\chi_{B}^{4}}{\chi_{B}^{2}}=1$. In HRG, and in the Boltzmann approximation, net-baryon kurtosis shows expected Skellam behavior [69,74]. The effect of repulsive interactions [second term in Eq. (29)] is to decrease this ratio. This is because the decrease of the susceptibility compared to ideal HRG due to the repulsive interaction increase with the order of the susceptibility. This behavior is also consistent with the lattice QCD data obtained in Refs. [94,99]. It was shown in Ref. [99] that at low temperature the ratio has a value unity and it decreases with temperature to reach the free quark limit at high temperature. Thus, our result, of MFHRG model, reproduces the lattice result. Deviation from the Skellam behavior can be attributed to the repulsive interactions between baryons, and again, we cannot neglect its contribution in the conserved charge fluctuation studies.

We next display the ratios for the electric charge susceptibilities in Fig. 4(b). Let us note that for electric charge susceptibilities, the mesons contribute dominantly and hence Boltzmann approximation will not be good approximation. Thus, simple expression similar to Eq. (29) cannot be obtained. Further, in the HRG model, only baryons with baryon number $B=1$ contribute to $\chi_{B}^{n}$, while in case of $\chi_{Q}^{n}$ multiple charged hadrons contribute. In fact, these multiply charged hadrons get larger weight in higher order fluctuations where both meson and baryons contribute. Upshot of this is the characteristic deviation of $\frac{\chi_{O}^{4}}{\chi_{Q}^{2}}$ seen in Fig. 4(b). The effect of repulsive interactions is to suppress the number density at high temperature. This can be seen in Fig. 4(b) as the ratio $\frac{\chi_{Q}^{4}}{\chi_{Q}^{2}}$ in MFHRG deviates from HRG model results. We have not displayed here any lattice results for the charge susceptibility ratio as the two susceptibilities, $\chi_{Q}^{2}$ and $\chi_{Q}^{4}$, are from different lattice simulation results, namely, $\chi_{Q}^{2}$ taken from Ref. [95] and $\chi_{Q}^{4}$ is taken from [96].

Finally, we show the similar ratio for the strangeness susceptibilities. The ratio of strangeness susceptibilities more or less gives a similar picture. Like the electric charge scenario, in the case of strangeness also the Boltzmann 

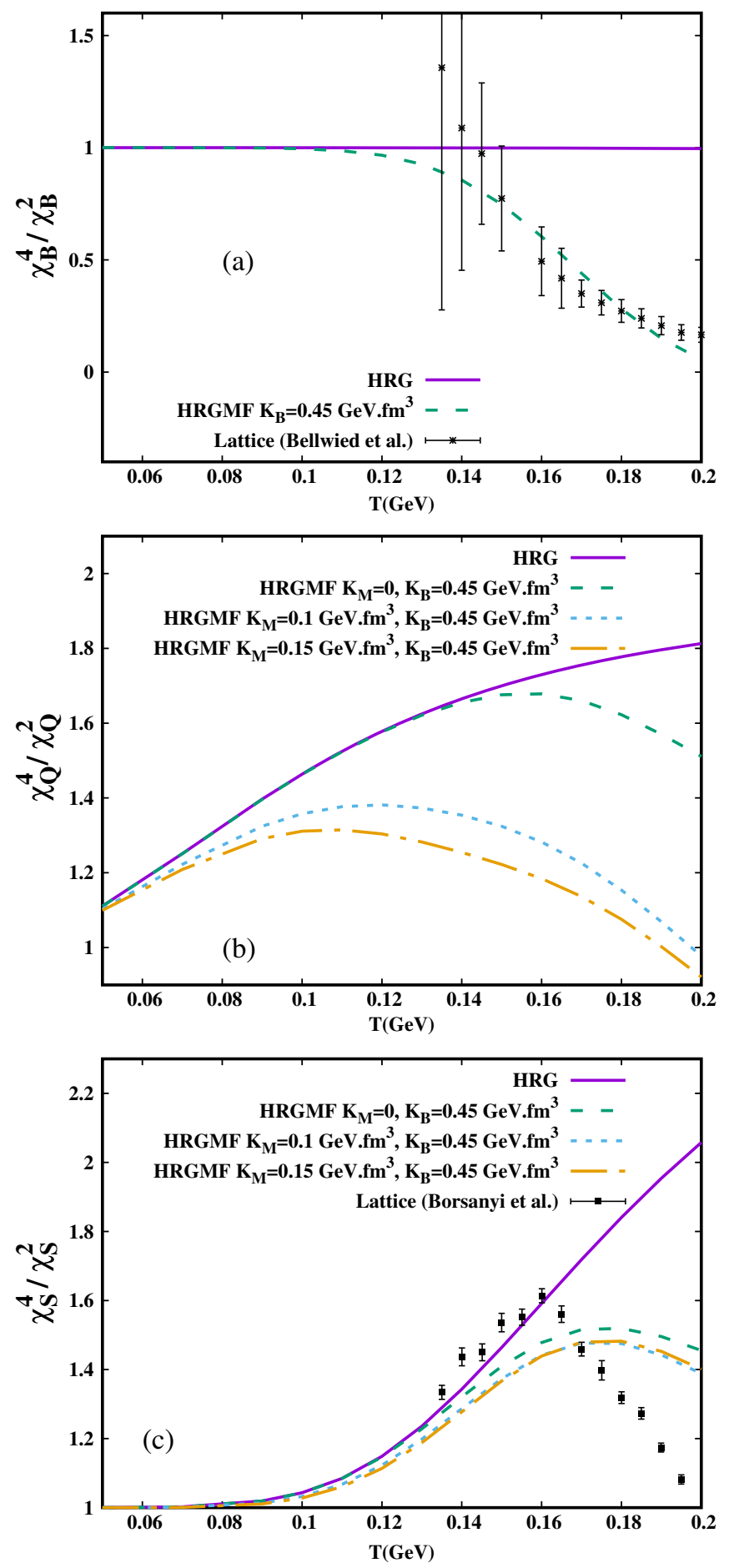

FIG. 4. Ratios of different order susceptibilities. The lattice data of $\chi_{B}^{4} / \chi_{B}^{2}$ are taken from [94] and the lattice data of $\chi_{S}^{4} / \chi_{S}^{2}$ are taken from [96].

approximation is not valid. Furthermore, the particles with multiple strangeness contribute to the scenario. For HRG, the ratio increases monotonically with temperature. Once we switch on the interaction, the ratio is suppressed. Furthermore, the ratio becomes nonmonotonic at high temperatures. The repulsive interaction seems to have a significant contribution in this quantity. Here again while the HRG results are closer to lattice for low temperatures, at high temperatures $(T \geq 160 \mathrm{MeV})$, HRG does not show the nonmonotonic behavior as the corresponding lattice results. On the other hand, MFHRG results display a qualitative similarity with nonmonotonic behavior with temperature as in lattice QCD results. In case of ratios of susceptibilities, it may not be easy to separate the effect of repulsive interactions from medium effects, like in-medium mass modification or the widening of spectral width. If one takes the difference of susceptibilities, the results are independent of mass spectrum included in the HRG model. Figure 5 shows differences of second and fourth order susceptibilities. The difference $\chi_{B}^{2}-\chi_{B}^{4}$ is zero in HRG model. But if we include the repulsive interactions using mean-field approach, then this difference increases with temperature. This behavior is in agreement with LQCD results [94]. The lattice result shows that the difference increases with temperature as has been found in the MFHRG model. The charge and strangeness sectors show different behavior as opposed to baryon sector in HRG model. Here, the differences of susceptibilities decrease with increase in temperature. This observation can again be attributed to multiple charged hadrons which contribute more to higher order susceptibilities. The effect of repulsive interactions is to suppress the heavier charged hadrons density at high temperature. Hence, we observe less steeper decrease in MFHRG as compared to HRG.

So far, we have shown the results for vanishing baryon chemical potential. Next, we discuss the case of nonvanishing values for the chemical potentials $\mu_{B}, \mu_{Q}$, and $\mu_{S}$. As shown in Ref. [85], in general, the susceptibilities at finite chemical potential can be expanded in powers of $\mu_{i} / T$ $(i=B, Q, S)$ with coefficients being generalized susceptibilities that can be evaluated at vanishing chemical potentials. The constraints of fixed strangeness and electric charge to baryon number fix the relation between the electric charge chemical potential and the strangeness chemical potential to the baryon chemical potential at a given temperature [100]. As in Ref. [85], we shall consider the case of strangeness neutral system i.e., $n_{S}=0$ and $n_{Q} / n_{B}=0.4$ which are representative conditions met in heavy-ion collision experiments with gold or uranium nuclei. Thus, one has the relation with $\hat{\mu}_{i}=\mu_{i} / T$,

$$
\begin{aligned}
& \hat{\mu}_{Q} \simeq q_{1}(T) \hat{\mu}_{B}+q_{3}(T) \hat{\mu}_{B}^{3} \\
& \hat{\mu}_{S} \simeq s_{1}(T) \hat{\mu}_{B}+s_{3}(T) \hat{\mu}_{B}^{3} .
\end{aligned}
$$

The coefficient functions $q_{i}$ and $s_{i}$ are given in terms of the susceptibilities at zero chemical potential and the ratio $r=n_{Q} / n_{B}$ as in Ref. [99]. We have calculated here these susceptibilities in MFHHRG model to calculate the coefficients on rhs of Eq. (30). Different susceptibilities are then estimated using Eq. (16) for a given $\mu_{B}, \mu_{Q}$, and $\mu_{S}$. 

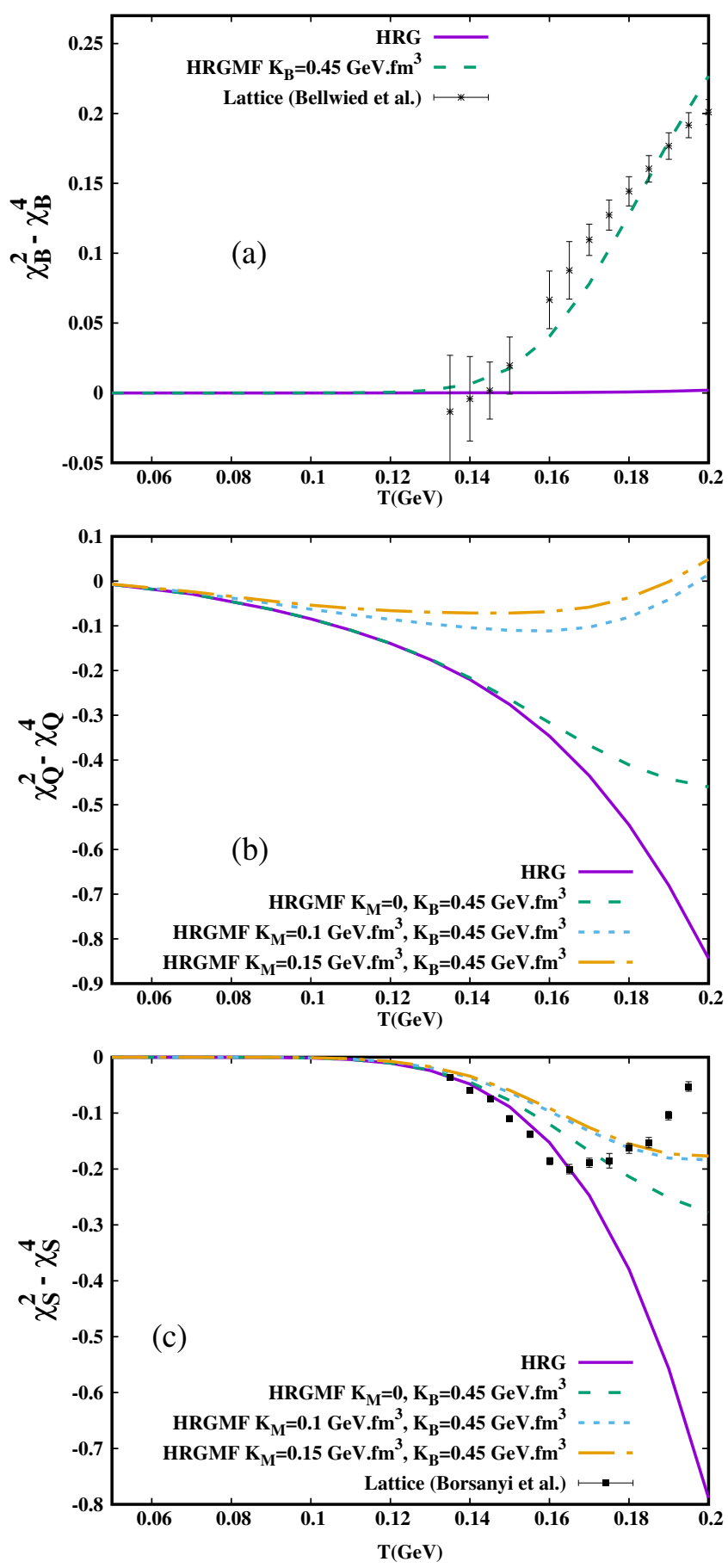

FIG. 5. Differences of second order and fourth order susceptibilities. The lattice data of $\chi_{B}^{2}-\chi_{B}^{4}$ are taken from [94] and the lattice data of $\chi_{S}^{2}-\chi_{S}^{4}$ are taken from [96].

In Fig. 6, we have plotted the ratio of the cumulants for the net baryon number fluctuations as functions of $\mu_{B} / T$. We have taken here, $T=158 \mathrm{MeV}$ as in Ref. [85] corresponding to the upper end of the error band of the pseudocritical temperature for vanishing chemical potential. On the top panel, we have plotted $R_{B}^{12}$, the ratio of mean to variance of net baryon number, i.e.,
$\chi_{B}^{1}\left(T, \mu_{B}\right) / \chi_{B}^{2}\left(T, \mu_{B}\right)$ as a function of $\mu_{B} / T$. On the middle panel, we have plotted the ratio of skewness ratio $R_{B}^{31}\left(T, \mu_{B}\right)=\frac{\chi_{B}^{3}\left(T, \mu_{B}\right)}{\chi_{B}^{1}\left(T, \mu_{B}\right)}$. Similarly, in the bottom panel, we have plotted the kurtosis ratio, i.e., $R_{B}^{42}=\frac{\chi_{B}^{4}\left(T, \mu_{B}\right)}{\chi_{B}^{2}\left(T, \mu_{B}\right)}$ as a function of $\mu_{B} / T$. As may be observed, the lower cumulant, i.e., $R_{B}^{12}$ from lattice simulations are in good agreement
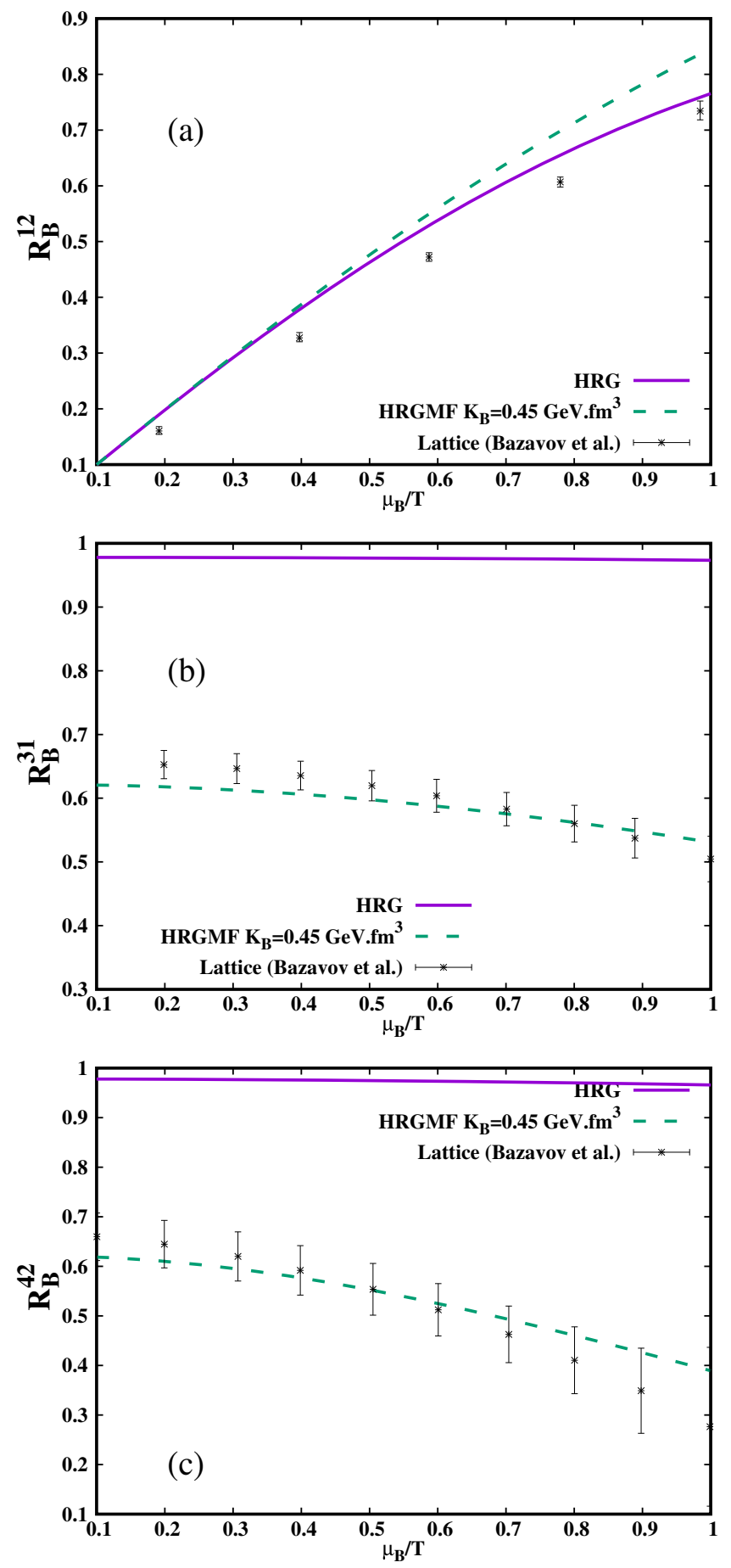

FIG. 6. $R_{B}^{12}, R_{B}^{31}$, and $R_{B}^{42}$ (defined in the text) as functions of $\mu_{B} / T$. The lattice data are taken from Ref. [85]. 
with the HRG model estimation which takes the hadrons to be pointlike and noninteracting. However, the simple HRG model is inadequate to describe the behavior of the higher order cumulants $R_{31}^{B}$ and $R_{42}^{B}$ obtained from lattice simulations. In fact, for simple HRG model, the values of both these higher order cumulant ratios are unity. When one includes the repulsive interactions among hadrons within MFHRG model, the lattice QCD results seem to be in agreement with the MFHRG model even at finite chemical potential. We have taken here the value of the baryonic repulsive parameter $K_{B}=0.45 \mathrm{GeV} \cdot \mathrm{fm}^{3}$ as was taken for zero baryon density case. Further, the baryonic susceptibilities are independent of $K_{M}$.

\section{SUMMARY}

We have studied the effect of repulsive interaction on the susceptibilities of conserved charges. For this purpose, we have used the MFHRG model. We have two parameters, namely, $K_{B}$ and $K_{M}$, to describe the baryonic and mesonic interactions, respectively. We have kept $K_{B}$ fixed at $0.45 \mathrm{GeV} \cdot \mathrm{fm}^{3}$ and varied $K_{m}$ in the range $0-0.15 \mathrm{GeV} \cdot \mathrm{fm}^{3}$. Our results have been confronted with the lattice data. We conclude the following important points:

(i) The results of HRG model, as such, cannot explain the LQCD data for baryonic susceptibilities. A repulsive interaction, through MFHRG model, is required to explain the data for the higher order susceptibilities. For low temperatures, HRG results for $\chi_{B}^{2}$ are somewhat closer to the lattice results as compared to MFHRG. However, for $\chi_{B}^{4}$, a repulsive mean field becomes a necessity to be consistent with the lattice QCD results for the same. The baryonic susceptibilities are independent of mesonic interactions. (ii) The repulsive interactions are further required to reproduce charge susceptibilities. The $\chi_{Q}^{2}$ is better reproduced with interactions compared to HRG. However, $\chi_{Q}^{4}$ is overestimated with the choice of the $K_{m}$ that we use though there is a qualitative matching. The trend shows a stronger mesonic interaction may reproduce data better.

(iii) The fourth order strangeness susceptibility is better reproduced, with the repulsive interactions, as compared to the second order susceptibility. However, calculation of strangeness susceptibilities have some inherent problems. The problem arises due to unknown strange hadronic states not included into the hadronic mass spectrum.

(iv) The ratios and differences of susceptibilities provide important information regarding interactions. These quantities provide us important details regarding the deviation from ideal gas scenario. Both the ratios and differences of susceptibilities are indicative of repulsive interaction of the hadrons. Such repulsive interaction, modeled by a repulsive mean field, as here, gives a reasonable description with the corresponding available lattice data particularly for those involving the higher order susceptibilities.

(v) The repulsive interactions for baryons become particularly important for finite density results. Our results, for the ratios of susceptibilities at finite density, have an excellent match with those obtained from the LQCD simulations.

\section{ACKNOWLEDGEMENT}

The authors gratefully acknowledge discussions with Prof. Prasad Hegde and Prof. Saumen Datta. G. K. was financially supported by DST-INSPIRE Faculty research Grant No. DST/INSPIRE/04/2017/002293. S. P. was financially supported by UGC, New Delhi.
[1] Y. Aoki, G. Endrodi, Z. Fodor, S. D. Katz, and K. K. Szabo, Nature (London) 443, 675 (2006).

[2] F. R. Brown, F. P. Butler, H. Chen, N. H. Christ, Z. Dong, W. Schaffer, L. I. Unger, A. Vaccarino et al., Phys. Rev. Lett. 65, 2491 (1990).

[3] S. Ejiri, Phys. Rev. D 78, 074507 (2008).

[4] R. D. Pisarski and F. Wilczek, Phys. Rev. D 29, 338 (1984).

[5] M. Asakawa and K. Yazaki, Nucl. Phys. A504, 668 (1989).

[6] M. A. Halasz, A. D. Jackson, R. E. Shrock, M. A. Stephanov, and J. J. M. Verbaarschot, Phys. Rev. D 58, 096007 (1998).

[7] Y. Hatta and T. Ikeda, Phys. Rev. D 67, 014028 (2003).
[8] E. S. Bowman and J. I. Kapusta, Phys. Rev. C 79, 015202 (2009).

[9] Z. Fodor and S. D. Katz, J. High Energy Phys. 04 (2004) 050 .

[10] M. A. Stephanov, Prog. Theor. Phys. Suppl. 153, 139 (2004).

[11] R. V. Gavai and S. Gupta, Phys. Rev. D 71, 114014 (2005).

[12] B. J. Schaefer and J. Wambach, Phys. Rev. D 75, 085015 (2007).

[13] R. V. Gavai and S. Gupta, Phys. Rev. D 78, 114503 (2008).

[14] M. Cheng et al., Phys. Rev. D 79, 074505 (2009). 
[15] G. Boyd, J. Engels, F. Karsch, E. Laermann, C. Legeland, M. Lugermeier, and B. Peterson, Nucl. Phys. B469, 419 (1996).

[16] J. Engels, O. Kaczmarek, F. Karsch, and E. Laermann, Nucl. Phys. B558, 307 (1999).

[17] Z. Fodor and S. D. Katz, Phys. Lett. B 534, 87 (2002).

[18] Z. Fodor, S. D. Katz, and K. K. Szabo, Phys. Lett. B 568, 73 (2003).

[19] C. R. Allton, S. Ejiri, S. J. Hands, O. Kaczmarek, F. Karsch, E. Laermann, Ch. Schmidt, and L. Scorzato, Phys. Rev. D 66, 074507 (2002).

[20] C. R. Allton, S. Ejiri, S. J. Hands, O. Kaczmarek, F. Karsch, E. Laermann, and Ch. Schmidt, Phys. Rev. D 68, 014507 (2003).

[21] C. R. Allton, M. Doring, S. Ejiri, S. J. Hands, O. Kaczmarek, F. Karsch, E. Laermann, and K. Redlich, Phys. Rev. D 71, 054508 (2005).

[22] P. de Forcrand and O. Philipsen, Nucl. Phys. B642, 290 (2002); B673, 170 (2003).

[23] Y. Aoki, Z. Fodor, S. D. Katz, and K. K. Szabo, Phys. Lett. B 643, 46 (2006).

[24] A. Bazavov et al., Phys. Rev. D 86, 034509 (2012).

[25] S. Borsányi, G. Endrődi, Z. Fodor, A. Jakovác, S. D. Katz, S. Krieg, C. Ratti, and K. K. Szabó, J. High Energy Phys. 11 (2010) 077.

[26] M. Cheng et al., Phys. Rev. D 77, 014511 (2008).

[27] S. Borsányi, Z. Fodor, S. D. Katz, S. Krieg, C. Ratti, and K. Szabó, J. High Energy Phys. 01 (2012) 138.

[28] C. Ratti, M. A. Thaler, and W. Weise, Phys. Rev. D 73, 014019 (2006).

[29] P. N. Meisinger and M. C. Ogilvie, Phys. Lett. B 379, 163 (1996).

[30] P. N Meisinger and M. C. Ogilvie, Nucl. Phys. B, Proc. Suppl. 47, 519 (1996).

[31] K. Fukushima, Phys. Lett. B 591, 277 (2004).

[32] E. Megies, E. R. Arriola, and L. L. Salcedo, Phys. Rev. D 74, 065005 (2006); 74, 114014 (2006).

[33] S. K. Ghosh, T. K. Mukherjee, M. G. Mustafa, and R. Ray, Phys. Rev. D 77, 094024 (2008).

[34] S. K. Ghosh, T. K. Mukherjee, M. G. Mustafa, and R. Ray, Phys. Rev. D 73, 114007 (2006).

[35] S. Mukherjee, M. G. Mustafa, and R. Ray, Phys. Rev. D 75, 094015 (2007).

[36] A. Bhattacharyya, P. Deb, S. K. Ghosh, and R. Ray, Phys. Rev. D 82, 014021 (2010).

[37] A. Bhattacharyya, P. Deb, A. Lahiri, and R. Ray, Phys. Rev. D 82, 114028 (2010).

[38] A. Bhattacharyya, P. Deb, A. Lahiri, and R. Ray, Phys. Rev. D 83, 014011 (2011).

[39] A. Bhattacharyya, P. Deb, S. K. Ghosh, R. Ray, and S. Sur, Phys. Rev. D 87, 054009 (2013).

[40] A. Bhattacharyya, S. K. Ghosh, S. Maity, S. Raha, R. Ray, K. Saha, and S. Upadhaya, Phys. Rev. D 95, 054005 (2017).

[41] A. Bhattacharyya, S. K. Ghosh, A. Lahiri, S. Majumder, S. Raha, and R. Ray, Phys. Rev. C 89, 064905 (2014).

[42] A. Bhattacharyya, S. K. Ghosh, S. Majumder, and R. Ray, Phys. Rev. D 86, 096006 (2012).

[43] P. Deb, A. Bhattacharyya, S. Datta, and S. K. Ghosh, Phys. Rev. C 79, 055208 (2009).
[44] P. Braun-Munzinger, K. Redlich, and J. Stachel, in Quark Gluon Plasma 3, edited by R. C. Hwa and X. N. Wang (World Scientific, Singapore, 2004).

[45] P. Braun-Munzinger, J. Stachel, J. P. Wessels, and N. Xu, Phys. Lett. B 344, 43 (1995).

[46] J. Cleymans, D. Elliott, H. Satz, and R. L. Thews, Z. Phys. C 74, 319 (1997).

[47] P. Braun-Munzinger, I. Heppe, and J. Stachel, Phys. Lett. B 465, 15 (1999).

[48] J. Cleymans and K. Redlich, Phys. Rev. C 60, 054908 (1999).

[49] F. Becattini, J. Manninen, and M. Gadzicki, Phys. Rev. C 73, 044905 (2006).

[50] P. Braun-Munzinger, D. Magestro, K. Redlich, and J. Stachel, Phys. Lett. B 518, 41 (2001).

[51] A. Andronic, P. Braun-Munzinger, and J. Stachel, Nucl. Phys. A772, 167 (2006).

[52] A. Andronic, P. Braun-Munzinger, and J. Stachel, Phys. Lett. B 673, 142 (2009).

[53] A. Bhattacharyya, S. K. Ghosh, R. Ray, and S. Samanta, Eur. Phys. Lett. 115, 62003 (2016).

[54] G. Kadam, S. Pal, and A. Bhattacharyya, J. Phys. G 47, 125106 (2020).

[55] B. J. Schaefer, J. M. Pawlowski, and J. Wambach, Phys. Rev. D 76, 074023 (2007).

[56] T. K. Herbst, J. M. Pawlowski, and B. J. Schaefer, Phys. Lett. B 696, 58 (2011).

[57] J. Wambach, B. J. Schaefer, and M. Wagner, Acta Phys. Pol. B Proc. Suppl. 3, 691 (2010).

[58] S. Chatterjee and K. A. Mohan, Phys. Rev. D 86, 114021 (2012).

[59] G. Ecker, Prog. Part. Nucl. Phys. 35, 1 (1995).

[60] J. Braun, W.-J. Fu, J. M. Pawlowski, F. Rennecke, D. Rosenblueh, and S. Yin, Phys. Rev. D 102, 056010 (2020); W. J. Fu, J. M. Pawlowski, and F. Rennecke, Phys. Rev. D 101, 054032 (2020); J. Braun, M. Leonhardt, and M. Poospiech, Phys. Rev. D 101, 036004 (2020).

[61] C. S. Fischer, Prog. Part. Nucl. Phys. 105, 01 (2019); F. Gao and J. M. Pawlowski, Phys. Rev. D 102, 034027 (2020); P. Isserstedt, M. Buballa, C. S. Fischer, and P. J. Gunkel, Phys. Rev. D 100, 074011 (2019).

[62] R. Dashen, S. K. Ma, and H. J. Bernstein, Phys. Rev. 187, 345 (1969).

[63] R. F. Dashen and R. Rajaraman, Phys. Rev. D 10, 708 (1974).

[64] G. M. Welke, R. Venugopalan, and M. Prakash, Phys. Lett. B 245, 137 (1990).

[65] R. Venugopalan and M. Prakash, Nucl. Phys. A546, 718 (1992).

[66] F. Karsch, K. Redlich, and A. Tawfik, Eur. Phys. J. C 29, 549 (2003).

[67] J. Noronha-Hostler, J. Noronha, and C. Greiner, Phys. Rev. Lett. 103, 172302 (2009).

[68] G. P. Kadam and H. Mishra, Nucl. Phys. A934, 133 (2014).

[69] V. Vovchenko, M. I. Gorenstein, and H. Stoecker, Phys. Rev. Lett. 118, 182301 (2017).

[70] F. Karsch and K. Redlich, Phys. Lett. B 695, 136 (2011). 
[71] P. Garg, D. K. Mishra, P. K. Netrakanti, B. Mohanty, A. K. Mohanty, B. K. Singh, and N. Xu, Phys. Lett. B 726, 691 (2013).

[72] S. Samanta, S. Chatterjee, and B. Mohanty, J. Phys. G 46, 065106 (2019).

[73] D. K. Mishra, P. K. Netrakanti, and B. Mohanty, Phys. Rev. C 94, 054906 (2016).

[74] M. Asakawa and M. Kitazawa, Prog. Part. Nucl. Phys. 90, 299 (2016).

[75] D. H. Rischke, J. Schaffner, M. I. Gorenstein, A. Schaefer, H. Stoecker, and W. Greiner, Z. Phys. C 56, 325 (1992).

[76] C. P. Singh, B. K. Patra, and K. K. Singh, Phys. Lett. B 387, 680 (1996).

[77] J. I. Kapusta and K. A. Olive, Nucl. Phys. A408, 478 (1983).

[78] K. A. Olive, Nucl. Phys. B190, 483 (1981).

[79] P. Huovinen and P. Petreczky, Phys. Lett. B 777, 125 (2018).

[80] G. Kadam and H. Mishra, Phys. Rev. D 100, 074015 (2019).

[81] A. Bazavov, H. T. Ding, P. Hegde, O. Kaczmarek, F. Karsch, E. Laermann, Y. Maezawa, S. Mukherjee, H. Ohno, P. Petreczky, C. Schmidt, S. Sharma, W. Soeldner, and M. Wagner, Phys. Rev. Lett. 111, 082301 (2013).

[82] M. I. Gorenstein, M. Hauer, and D. O. Nikolajenko, Phys. Rev. C 76, 024901 (2007).

[83] J. Fu, Phys. Lett. B 722, 144 (2013).

[84] A. Bazavov et al., Phys. Rev. Lett. 109, 192302 (2012); C. Schmidt (for the BNL-Bielefeld Collaboration), Proc. Sci. ConfinementX2012 (2012) 187; A. Bazavov et al., Phys. Rev. Lett. 111, 072001 (2014); 113, 072001 (2014).
[85] A. Bazabov et al., Phys. Rev. D 101, 074502 (2020).

[86] S. Jeon and V. Koch, Phys. Rev. Lett. 83, 5435 (1999).

[87] S. Jeon and V. Koch, Phys. Rev. Lett. 85, 2076 (2000).

[88] A. Bhattacharyya, S. Das, S. K. Ghosh, R. Ray, and S. Samanta, Phys. Rev. C 90, 034909 (2014).

[89] A. Bhattacharyya, R. Ray, and S. Sur, Phys. Rev. D 91, 051501 (2015).

[90] A. Bhattacharyya, R. Ray, S. Samanta, and S. Sur, Phys. Rev. C 91, 041901 (2015).

[91] A. Bhattacharyya, S. K. Ghosh, S. Maity, S. Raha, R. Ray, K. Saha, S. Samanta, and S. Upadhaya, Phys. Rev. C 99, 045207 (2019).

[92] A. Bhattacharyya, S. K. Ghosh, R. Ray, K. Saha, and S. Upadhaya, Europhys. Lett. 116, 52001 (2016).

[93] M. Tanabashi et al. (Particle Data Group), Phys. Rev. D 98, 030001 (2018).

[94] R. Bellwied, S. Borsanyi, Z. Fodor, S. D. Katz, A. Pasztor, C. Ratti, and K. K. Szabo, Phys. Rev. D 92, 114505 (2015).

[95] M. Sarkar, O. Kaczmarek, F. Karsch, A. Lahiri, and C. Schmidt, Proc. Sci. LATTICE2019 (2019) 087.

[96] S. Borsanyi, Z. Fodor, J. N. Guenther, S. K. Katz, K. K. Szabo, A. Pasztor, I. Portillo, and C. Ratti, J. High Energy Phys. 10 (2018) 205.

[97] A. Bazavov et al., Phys. Rev. Lett. 113, 072001 (2014).

[98] P. M. Lo, M. Marczenko, K. Redlich, and C. Sasaki, Phys. Rev. C 92, 055206 (2015).

[99] A. Bazavov et al., Phys. Rev. D 95, 054504 (2017).

[100] A. Bazabov et al., Phys. Rev. D 96, 074510 (2017). 